\title{
Erratum to: Factors influencing network risk judgments: a conceptual inquiry and exploratory analysis
}

Jennifer Cowley ${ }^{1 *}$, Frank L Greitzer ${ }^{2}$ and Bronwyn Woods ${ }^{1}$

\section{Erratum}

The original published version of this article [1] stated that the received date of the original manuscript was 4 April 2010; however, the original manuscript was actually received on 4 April 2014.

\section{Author details \\ ${ }^{1}$ Carnegie Mellon University Software Engineering Institute, CERT Division, 4500 Fifth Avenue, Pittsburgh, Pennsylvania 15213, USA. ${ }^{2}$ PsyberAnalytix, 651 Big Sky Drive, Richland, Washington 99352, USA.}

Received: 6 May 2015 Accepted: 6 May 2015

Published online: 22 May 2015

\section{Reference}

1. J Cowley, FL Greitzer, B Woods, Factors influencing network risk judgments: a conceptual inquiry and exploratory analysis. Secur. Inform. 4, 1 (2015)

\footnotetext{
*Correspondence: jcowley@cert.org

${ }^{1}$ Carnegie Mellon University Software Engineering Institute, CERT Division, 4500 Fifth Avenue, Pittsburgh, Pennsylvania 15213, USA

Full list of author information is available at the end of the article
}

Submit your manuscript to a SpringerOpen ${ }^{\odot}$ journal and benefit from:

- Convenient online submission

- Rigorous peer review

- Immediate publication on acceptance

- Open access: articles freely available online

- High visibility within the field

- Retaining the copyright to your article

Submit your next manuscript at $>$ springeropen.com 